\title{
Fundamentals of Membrane Transport
}

\author{
H. L. FRISCH \\ Department of Chemistry, State University of New York at Albany, \\ 1400 Washington Avenue, Albany, NY 12222 \\ (Received November 26, 1990)
}

\begin{abstract}
Mass transport through thermally activated molecular dispersal (diffusion) in multicomponent mixtures comprising membrane systems can exhibit complexities which require different levels of physical and theoretical descriptions of which we give examples. At the simplest level mean field level component flows are derived from gradients of chemical potential. We will focus primarily in our talk on the mean field description in homogeneous or inhomogeneous polymer systems. Penetrant diffusion in homogeneous "rubbery" polymer films or membranes can be relatively successfully described by a simple "free volume" theory. Inhomogeneities due to e.g. polymer crystallinity are a further complication and are an essential aspect in diffusion in very porous systems such as rigid polymer foams. Further complications arise in mechanically relaxing polymer films with memory which are not in mechanical equilibrium. Such effects as well as penetrant stress driven diffusion and dual mode sorption can arise in polymer penetrant systems below their glass transition temperature.
\end{abstract}

Key Words: Membrane Transport/Diffusion/Sorption/ Polymer Films / 
Mass transport through thermally activated molecular dispersal (diffusion) in multicomponent mixtures comprising membrane systems can exhibit complexities which require different levels of physical and theoretical description. If not only the average number density of diffusing particle species but also their local fluctuations (in space and time) play a significant role in specifying the dynamics of diffusion then the system has to be described at least at the level of a statistical mechanical master equation 1 . We give two examples of such situations: First this occurs in the diffusion of molecules along the contours of a few membrane macromolecules whose hopping is aided by rapidly fluctuating cofactors with which the molecules have to cooperatively bind at saturating sites of the macromolecules. A second much recently studied ${ }^{2-3}$, example is afforded by diffusion-limited reaction processes. Fluctuations play an important role because fast reactions imply that particles react mostly with their immediate neighbors. 2,3 As a result "mean field" hydrodynamic equations specifying the local mean concentration changes expressed as a sum of one term describing changes due to diffusion and another due to the chemical reactions fail to apply. Under certain initial and boundary conditions this failure is difficult to see (as numerical simulations show) if one follows a single macroscopic parameter-such as the total flow up to time $t$ of the membrane of reactants - for permeation in a membrane subject to strongly diffusion controlled non-linear reaction kinetics ${ }^{4}$. In particular entities, satisfying particle conservation, like the time lag, $\mathrm{L}$, to achieve steady state permeation are universal ${ }^{4}$ ( $i . e$. depend only on initial and boundary conditions but not on details of the progress of the chemical reaction).

The simplest level of a "mean field" description fortunately suffices in many instances. 5 When the systems are in mechanical equilibrium as a whole ${ }^{5}$ the various component flows can be derived from the gradients of chemical potentials (in electrically charged systems the gradients of the electrochemical potentials) which can also reflect temperature, pressure, etc. gradients if these are present. Interface effects can be relegated at this level to suitable boundary conditions. "Faciliated diffusion" requires a multiplicity of vectorial flows of species and carrier species in a membrane ${ }^{6}$. Care must be exercised in specifying the various coupled flows and fluxes taking proper account of the frame of reference particularly in systems which swell and exhibit significant volume changes on mixing.5,7 Coupling of diffusive flows with sufficiently "slow" chemical kinetics can occur and can sometimes be treated adequately phenomenologically at a mean field, "hydrodynamical" level as in theories of "active transport". 6

To review the whole "mean field" theory of diffusion in all membranes is outside the scope of a single paper but we will highlight many concepts and theories of the diffusive transport of small molecules (which we call penetrants) in homogeneous (and to a lesser extent inhomogeneous) polymer membranes and films. In the next section we will briefly classify the varieties $8-11$ of penetrant diffusion behavior in polymers within the context of their almost ubiquitous experimental measurement. 8 succeeding sections deal with specialized topics such as certain diffusion anomalies and their description at or below the glass transition temperature, $\mathrm{Tg}$, of the polymer-penetrant system and the outline of "free volume" theory $8,9,12,13$ of diffusion which semi-quantitatively correlates diffusion behavior in systems above $\mathrm{Tg}$. 
We will also briefly describe limiting situations when the very singular geometry of some heterogeneities produce new diffusion effects.

Measurement and classification of diffusion in polymer membranes

a. Ideal diffusion

Permeation of simple gases through thin polymer membranes (natural rubber), of thickness $\ell$, was already studied by Graham ${ }^{14}$ a hundred and twenty-five years ago. Gases such as nitrogen at one atmosphere or less of partial pressure at room temperature dissolve or more correctly are sorbed, forming a very dilute penetrant polymer solution at the high pressure reservoir side (at partial pressure $p$ in Figure 1) of the membrane, diffuse molecularly to the low pressure side (partial pressure $p=0$, for convenience at $x=\ell$ ) and evaporate at that surface into the adjacent reservoir. Also shown in Figure 1 is the total flow up to time $t$ of gas per unit membrane area out of the membrane as a function of $t$. The rate of total flow increases steadily until a constant steady-state value, $\mathrm{J}_{S}$, (the derivative of the linear portion of the curve in Figure 1 ) is achieved. $J_{s}$ is the steady state flux (amount of gas per unit area and time) out of the membrane at $x=\ell$.
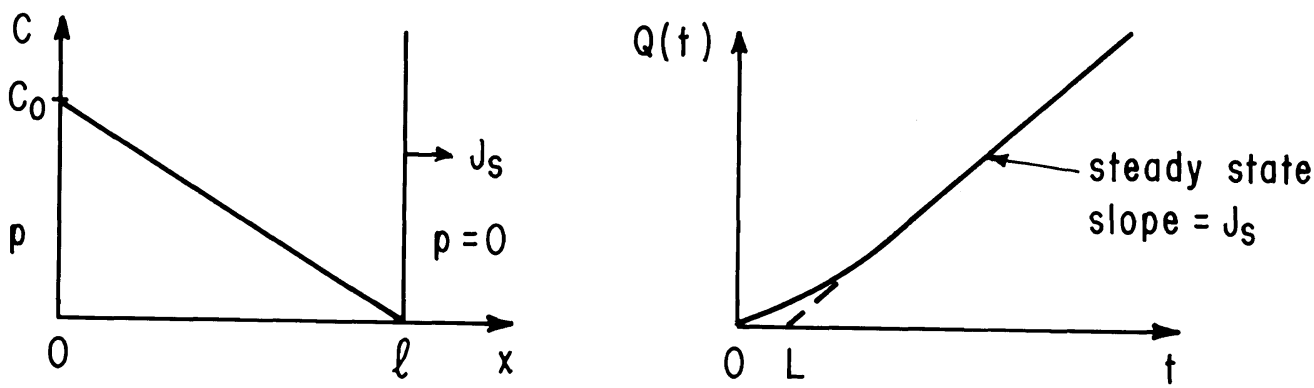

Figure 1. Permeation cell experiment

The quantitative relationships underlying such a permeability-cell measurement were formulated over a hundred years ago by Von Wroblewski. 15 polymers in the rubbery state respond very rapidly to changes in their condition, hence mechanical equilibrium is maintained, and sorption equilibrium is achieved almost instantaneously as in a low molecular weight liquids. Von Wroblewski realized first that the solubility of the gas is proportional to the partial pressure above the polymer membrane (a consequence of Henry's Law)

$$
c(x=0, t)=c_{0}=k_{D} p ; c(x=l, t)=0
$$

with $k_{D}$ the constant Henry's Law solubility coefficient and secondly that at steady state Fick's First Law of diffusion (flux is the product of the diffusion coefficient times the negative concentration gradient) implied the linear law

$$
\mathrm{J}_{\mathrm{s}}=\mathrm{D}_{0} \mathrm{C}_{\mathrm{o}} / \ell
$$


This could be combined with eq. (1) to obtain the we11-known permeability equation 8

$$
J_{S}=D_{O} k_{D}(p / \ell)=P(p / \ell),
$$

with $P$ the permeability constant.

Eq. (2) is the basis of the definition of one of the fundamental diffusion coefficients obtainable from steady-state diffusive transport, the effective diffusion coefficient $D_{\text {eff }}$

$$
D_{\text {eff }}=J_{s} /\left(c_{0} / \ell\right)
$$

which in our special case of ideal or Case I diffusion is given by the constant $D_{o}$. Since in the usual permeability cell measurement $c_{o}$ is not known one can use the asymptote to the time axis of the flow, the time lag, L, shown in Figure 1 as a measure of the time taken to achieve a steady state of permeation. From dimensional analysis alone it is clear that (for ideal diffusion) $L$ must be proportional to $\ell^{2} / D_{0}$ and Daynes 16 showed (for our boundary conditions) that

$$
L=\ell^{2 / 6 D_{O}} \text {. }
$$

In the hands of Barrer 17 and his successors this became an important method of defining another diffusion coefficient, the time lag diffusion coefficient, $\mathrm{D}_{\mathrm{L}}$

$$
D_{L}=\ell^{2 / 6 L}
$$

which for ideal or Case I systems has the constant value, $D_{0}$, just like Deff.

Diffusion coefficients can also be defined by sorption studies 7,8 into thin membranes of thickness $\ell$ and $f$ ixed area. Even lower molecular weight vapors, at room temperature, but sufficiently low partial pressure will be sorbed into initially degassed rubbery polymer membranes to form after sufficiently long times very dilute penetrant-polymer solutions. In that case the relative weight uptake $M_{t} / M_{\infty}$ ( $M_{t}$ weight change up to time $t, M_{\infty}$ that after infinite time) when plotted versus $t^{1 / 2 / l}$ is a curve saturating in time shown in Figure 2 with a considerable initial portion whose slope $R_{a}$ also defines a diffusion coefficient, $D_{a}$

$$
\mathrm{D}_{\mathrm{a}}=(\pi / 16) \quad \mathrm{R}_{\mathrm{a}}^{2}
$$

Another diffusion coefficient can be defined by the equivalent initial slope for the desorption of such a membrane into vacuum, $R_{S}$, via

$$
D_{S}=(\pi / 16) R_{S}^{2} .
$$


For ideal or Case I diffusion of course Figure 2 also gives the desorption relative weight loss and so as expected $D_{a}=D_{s}=D_{0}$.

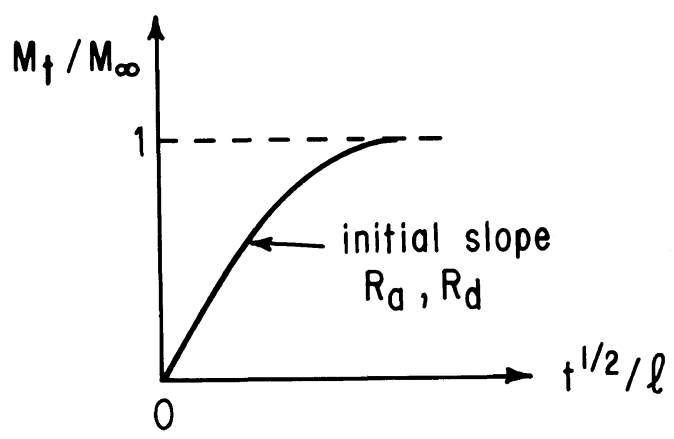

Figure 2. Sorption-desorption weight uptake curve

Concentration distance curves 7,8 at fixed time $t$ of a penetrant diffusing essentially one dimensionally into a sufficiently thick slab of polymer whose surface at $x=0$ is maintained at the fixed penetrant concentration $c_{0}$ provides another diffusion coefficient. Choosing an experimentally convenient concentration $c^{\prime}, c_{0}>c^{\prime}>0, D_{x}\left(c^{\prime}\right)$, is found by introducing Boltzmann's transformation ${ }^{7}$ into Fick's Second Law of Diffusion and solving for the diffusion coefficient

$$
D_{x}\left(c^{\prime}\right)=-(1 / 2 t)(d x / d c) c^{\cdot} \int_{0}^{c^{\prime}} x d c \text {, }
$$

and yields again for ideal diffusion the same constant, $D_{\mathbf{x}}\left(c^{\prime}\right)=D_{0}$.

b. Fickian concentration dependent diffusion and effects due to heterogeneity.

The fact that these different methods of measuring the diffusion coefficient yield the same number $D_{0}$ is a consequence of the very dilute (Henry's Law 1imit) penetrant-rubbery polymer solution which is being studied. Thus returning to our previous example of nitrogen diffusing at room temperature in natural rubber at an applied partial pressure $p$ - if that pressure is raised from about one atmosphere to several hundred atmospheres the resulting solution is no longer very dilute. As a consequence the solubility coefficient $k_{D}$ becomes a function of $p$ and the diffusion coefficient $D(c)$ becomes a function of the local concentration which varies with position in the membrane. There can be several causes ${ }^{8}$ for this concentration dependence of $D$ : In protein membranes in which acidic penetrants diffuse (particularly in the presence of some little water vapor) the diffusion occurs from sites at which the penetrants are relatively strongly bound. Thus at small concentrations where the sites are saturated by previously diffused penetrant the diffusion coefficient can, at least initially, decrease 
with concentration. Also, particularly with organic penetrants, in organic, rubbery polymers (even at partial pressures below one atmosphere) non-dilute solutions are formed in which the segmental mobility of the polymer is enhanced by "plasticization" due to the organic penetrant. This produces strikingly large increases in $D(c)$ with increasing $c$ which we will further discuss later under the context of "free volume" theories of diffusion. For partial pressures of only a few atmospheres, a rough engineering measure of whether or not a limiting very dilute penetrant-polymer solution is formed is given by the critical temperature, $T_{c}$, of the penetrant. Thus if $T$ is the ambient temperature at which the transport process is carried out a value $T>T_{c}$, suggests that a very dilute solution is attained in which Case I or ideal diffusion is observed. If on the other hand $T \leq T_{c}$ then significant departures from ideal diffusion are generally observed.

Another set of complications arises because real polymer membranes are accidentally or with intention inhomogeneous. 7,8 These inhomogeneities arise due to domains of phase separation in polymer blends or block copolymers, crystalline regions (with highly restricted access of the penetrant) in crystalline polymers and fillers which might be added to strengthen the membrane, etc. Providing the differential geometry of these domains is sufficiently smooth and we are dealing with systems above their glass transition temperature under conditions of mechanical equilibrium and under experimental circumstances in which there are no gradients in temperature or pressure (i.e. $T$ and $p$ are fixed) we can often describe diffusive transport with two functions of position $\vec{r}$ and concentration $c$. These are the penetrant component mobility relative to the polymer component, $m_{d}(\vec{r}, c)$, and the thermodynamic partition coefficient, $k(\vec{F}, c)$, which is the reciprocal of the local activity coefficient, i.e. activity $=a=c / k$. Both are also functions of $T$ and $p$. Under these conditions relaxation effects and their memory, penetrant stress effects, flow, and inertial effects are negligible. The local chemical potential of the (uncharged) penetrant, $\mu$, can be given by its instantaneous value at each location $\vec{r}$, ( $R$ gas constant)

$$
\mu=\mu^{\circ}(T)+\operatorname{RT} \ln [c / k(\vec{r}, c)],
$$

with $\mu^{\circ}(T)$ determined only by the reference standard state. The vectorial flux of penetrant $\vec{J}$ at $\vec{r}$ and time $t$ is given by nonequilibrium thermodynamics 5 by the generalization of Fick's First Law

$$
\vec{J}=-m_{d}(\vec{r}, c) c \operatorname{grad} \mu,
$$

where the coefficient of grad $\mu$ is the single onsager coefficient 5 necessary to specify the flow in this "two component" penetrant polymer system. In a region devoid of sources or sinks of penetrant we also have penetrant mass conservation (essentially Fick's Second Law)

$$
\frac{\partial c}{\partial t}=-\operatorname{div} \overrightarrow{\mathrm{J}} \text {. }
$$

Combining eqs. (10)-(12) yields a diffusion equation, which has actually the form of a Fokker-Planck equation ${ }^{1}$ ( $k$ being given by equilibrium statistical mechanics and is such that microscopic reversibility is maintained) 


$$
\frac{\partial c}{\partial t}=\operatorname{div}\left\{D_{T}(\vec{r}, c) k(\vec{r}, c) \operatorname{grad}[c / k(\vec{r}, c)]\right\}
$$

with the thermodynamic diffusion coefficient 9

$$
D_{T}(\vec{r}, c)=\operatorname{RTm}_{d}(\vec{r}, c) .
$$

Two simplifications of eq. (13) arise in the absence of explicit inhomogeneities but in more concentrated systems with concentration dependence when $k=k(c), m_{d}=m_{d}(c), D_{T}=D_{T}(c)(g r a d ~ c / k(c)=$ $d(c / k) / d c$ grad $c)$ and (13) becomes in thin films or membranes where diffusion is one dimensional (say along the $x$ axis, $0<x<l$ )

with

$$
\frac{\partial c}{\partial t}=\frac{\partial}{\partial x}\left[D(c) \frac{\partial c}{\partial x}\right]
$$

$$
D(c)=D_{T}(c) \quad\left[1-\frac{d \ell n k}{d \ell n c}\right] .
$$

Such systems are called "Fickian" if sorption equilibrium is attained at the boundaries, $9,18 \mathrm{e} . \mathrm{g}$. in a permeation cell $c(x=0, t)=c_{0}$, $c(x=l, t)=0$ and $c(x, 0)=0$. Under these conditions if one measures various diffusion coefficients using eqs. (4), (6)-(9) one obtains different functionals of the concentration dependent diffusion coefficient $D(c)$, all functions of $c_{0}$. Thus as shown by Barrer 17 the effective diffusion coefficient is obtained by direct quadrature of the steady state version of eq. (15) yields the mean diffusion coefficient $D\left(c_{0}\right)$, (cf. eq. (4))

$$
D_{\text {eff }}\left(c_{0}\right)=J_{s} /\left(c_{0} / \ell\right)=D\left(c_{0}\right)=\int^{c} o_{D}(c) d c / c_{0} .
$$

The time $1 \mathrm{ag}$ diffusion coefficient is (obtained 19 from a conservation condition involving only the steady state concentration and $D(c)$ )

$$
D_{L}\left(c_{0}\right)=l^{2 / 6 L}=\frac{1 / 6\left[\int D(c) d c\right]^{3}}{\int_{0}^{C_{0} d w w D(w)} \int_{w}^{c_{0}} D(u) d u}
$$

Even for physically realistic but highly restricted $D(c)$ these two diffusion coefficients can be significantly different - e.g. if $D(c)$ is a given by power series with positive coefficients or $\ln \int D(u) d u$ is a convex function of $c_{0}$ then

$$
1 \leq \mathrm{D}_{\mathrm{eff}} / \mathrm{D}_{\mathrm{L}} \leq 3
$$

From extensive numerical simulations, Crank ${ }^{7}$ recommends the empirical "weighted mean" expressions for $D_{a}\left(c_{0}\right)$ and $D_{x}\left(c_{0}\right)$

$$
D_{a}\left(c_{0}\right)=(\pi / 16) R_{a}^{2}\left(c_{0}\right) \simeq p c_{0}^{-p} \int_{0}^{c_{0}} c^{p-1} D(c) d c
$$

with $1.62<p<1.75$ and "best" $p=1.67(=5 / 3)$ and

$$
D_{S}\left(c_{0}\right)=(\pi / 16) R_{S}^{2}\left(c_{0}\right) \simeq q c_{0}^{-q} \int_{0}^{c_{0}}\left(c_{0}-c\right) q-1 D(c) d c
$$


with $1.72<\mathrm{q}<1.89$ and "best" $q=1.85$. The concentration-distance

diffusion coefficient ${ }^{7}$ is

$D_{x}\left(c^{\prime}\right)=-(1 / 2 t)(d x / d c) c^{\prime} \int_{0}^{\prime} x d c=D\left(c^{\prime}\right)$.

Clearly different methods of measuring diffusion coefficients yield now

different values - just as in analogy-different ways of measuring the mean molecular weight of polydisperse polymers define different

molecular weight averages. We have not commented on frame of reference corrections. In swelling thin polymer membranes in the $\mathbf{x}$ direction (measured from the edge of the sample) the concentration $c$ should be chosen to be the volume fraction of the polymer and $D(c)$ is then the polymer fixed binary diffusion coef ficient. $7,8,12,13,17$

For explicitly inhomogeneous polymer membranes without concentration dependence (ideal diffusion) can be described by functions of location, $r, k=k(r)$ and $D_{T}(r)=D(r)$. These are really stochastic variables whose distribution is rarely known with any confidence. In many standard crystalline polymers the simplest statistical parameter the volume fraction of crystalline polymer, $\phi_{c}$ can differ easily by more than $5 \%$ when determined by different methods - density, $X$-ray or infra-red. In any case eq. (13) now reduces to

$$
\frac{\partial c}{\partial t}=\operatorname{div}\{D(\vec{r}) k(\vec{r}) \operatorname{grad}[c / k(\vec{r})]\}
$$

Even the steady-state solution of this equation has resisted all efforts except for specialized cases $7,8,4$ of regular periodic arrays, very dilute dispersions of one convex shape or lamellar media where $k=k(x)$ and $D=D(x)$, etc. Thus $D_{\text {eff }}$ is only known in these special cases more generally there are strict bounds and various variational approximations 20,21 for $D_{\text {eff }}$ and the time lag $\mathrm{L}$ is a known functional of the steady state concentration 22 . Using a short time eikonal asympototic development of the Laplace transform of the solution of eq. (22) one finds ${ }^{23}$ that for initial sorption into a slab of volume $v$ with plane faces at $x=0, l$ one obtains instead of (7) or (19)

$$
D_{a}=\left\{\frac{1}{2}\left[\frac{\left\langle k^{1 / 2}(\vec{r}) D^{-1 / 4}(\vec{r})>_{0}\right.}{\left\langle\left(D^{3 / 4}(\vec{r}) k^{1 / 2}(\vec{r})\right)^{-1}>_{0}\right.}+\frac{\left\langle k^{1 / 2}(\vec{r}) D^{-1 / 4}(\vec{r})\right\rangle_{\ell}}{\left\langle\left(D^{3 / 4}(\vec{r}) k^{1 / 2}(\vec{r})\right)^{-1}\right\rangle_{\ell}}\right]\right\}^{2}
$$

where $\langle\ldots\rangle_{0, l}$ is the surface average over the surface $x=0$ or $\ell$ and $\langle\ldots\rangle_{v}$ is the volume average. Numerous results in special heterogeneous model media have been obtained 7,8 by Barrer, Ash, Barrie, Petropoulos, Roussis, Grzywna, Crank, etc. The main point is that in heterogeneous media different experimental diffusion coefficient measurements can result in widely different values.

The temperature dependence of diffusion of gases and vapors in rubbery polymers at temperatures far from $\mathrm{Tg}$ satisfies ${ }^{8}$ an apparent Arrhenius dependence for Case I diffusion but in more concentrated penetrant-polymer solutions one sees often departures from the constancy of the diffusion activation energy as the glass transition temperature is approached ${ }^{9}$.

Table I summarizes the diffusion behavior of penetrants in polymer membranes $7,8,11$. 
Table I

Diffusion Behavior of Penetrants in Homogeneous Polymer Membranes

\begin{tabular}{|c|c|c|}
\hline & $\mathrm{T}>\mathrm{Tg}$ & $\mathrm{T}<\mathrm{Tg}$ \\
\hline $\mathrm{T}>\mathrm{T}_{\mathrm{c}}$ & $\begin{array}{l}\text { Ideal or Case I diffusion } \\
k_{D} \text { independent of } p \\
D=D_{0} \text { independent of } \\
\text { concentration. } \\
\text { For } T>T g \text {, Arrhenius } \\
\text { temperature dependence } \\
\text { of } D\end{array}$ & Dual Mode Sorption \\
\hline $\mathrm{T}<\mathrm{T}_{\mathrm{c}}$ & $\begin{array}{l}\text { Concentration dependent } \\
\text { diffusion } D=D(c) \\
k_{0} \text { can depend on } p . \\
\text { Sorption equilibrium } \\
\text { generally attained } \\
\text { at boundaries. } \\
\text { Fickian diffusion } \\
\text { Free volume behavior }\end{array}$ & $\begin{array}{l}\text { Memory effects and } \\
\text { "time dependent" diffusion. } \\
\text { Diffusion anomalies in } \\
\text { sorption and permeation } \\
\text { Concentration dependent } \\
\text { dual mode sorption } \\
\text { Crazing and mechanical } \\
\text { failure of polymers in } \\
\text { which diffusion occurs. }\end{array}$ \\
\hline
\end{tabular}

c. Diffusion in glassy polymers

Diffusion in glassy polymers involves complex phenomena which are incompletely understood $7-9.11,12,13,24$. Just below the glass transition for a penetrant-polymer system (whose $\mathrm{Tg}$ differs at least somewhat from that of the pure polymer and depends on concentration) the segmental micro-Brownian motion of at least some small fraction of the polymer chains is drastically slowed down. As the temperature is further reduced an increasing fraction of the chains in the sample have backbone micro-Brownian motion which is "frozen" out. This has at least two consequences for the sorption of another penetrant molecule and its molecular dispersal below the $\mathrm{Tg}$ of the system. The penetrant molecule can be sorbed into a volume region, large enough to accommodate it, surrounded relatively intimately, primarily by chain segments of chains whose micro-Brownian motion is not frozen out or it can be surrounded by segments of a majority of chains whose backbone motion is frozen out. The absence of a classical isotope effect for penetrants even as small as the hydrogen isotopes 25 suggests that no free penetrant center of mass motion occurs even above $\mathrm{Tg}$ but that penetrant motion is mechanically coupled directly to that of the chain segments surrounding the penetrant. 13 The penetrant surrounded and moved along by segments of chains whose backbone micro-Brownian motion is not frozen out is by virtue of the considerable segment mobility of these chains much more mobile than the penetrant molecules surrounded by segments stemming from chains whose backbone is frozen out. We can speak of a "rubber" segment environment and one (or more) "glassy" segment environments of a penetrant. Probably finer future experimental techniques will reveal a multiplicity of "glassy" segment environments depending on the fraction 
of more or less immobilized segments surrounding a penetrant in such an environment. But for the present simplifying to only one such "glassy" environment we have the basis of seeing different sorption behavior dual mode sorption.13,24,26 In one case we have sorption involving relatively rapidly moving sites (Henry's Law sorption in a dilute system) due to the large unfrozen chain segment mobility surrounding the penetrant. In the other case we have sorption into relatively immobile sites, which can saturate, and can thus be approximately described by a Langmuir isotherm. The situation is complicated by the second consequence that restricted mobility of both chain segments and penetrant motion prevents rapid establishment of equilibrium chain configurations throughout the sample. Thus the observation of dual mode sorption below $\mathrm{Tg}$ can be mitigated by the thermo-mechanical history of the sample. Further observed complications require augmentation of the diffusion equations by terms arising from mechanical relaxation effects or penetrant stress driven effects $27-29$. These questions will be more fully considered in my talk. Not only new physical techniques but our ability to numerically simulate diffusion in polymers 30 will help elucidate this field.

\section{Acknowledgement}

This work was supported by the National science Foundation.

\section{References}

1. N.G. Van-Kampen, "Stochastic Processes in Physics and Chemistry", North-Holland, Amsterdam, 1981.

2. D. Toussaint and F. Wilczek, J. Chem. Phys. 78, 2642 (1983); K. Kang and S. Redner, Phys. Rev. A 32, 435 (1985); L.W. Anacker and R. Kopelman, Phys. Rev. Lett. 58,289 (1987), K. Lindenberg, B.J. West and R. Kopelman, ibid. 60,1777 (1988).

3. C.R. Doering and D. ben-Avraham, Phys. Rev. A 38, 3035 (1988), ibid. A 37, 5007 (1988), Phys. Rev. Lett. 62, 2563 (1989).

4. D. ben-Avraham and H.L. Frisch, Phys. Rev. A 40, 505 (1989).

5. D.H. Fitts, "Nonequilibrium Thermodynamics", McGraw Hill, New York, 1962; S.R. de Groot and P. Mazur, "Non-Equilibrium Thermodynamics:, North Holland, Amsterdam, 1969; E.L. Cussler, "Multicomponent Diffusion", Elsevier Scientific, New York, 1976.

6. M. Cereido and C.A. Rotunno, "Introduction to the Study of Biological Membranes", 2० Edit., Gordon and Breach, New York, 1971; 0. Kedem and A. Katchalsky, J. Gen. Physio1. 45, 143 (1961), Trans. Faraday Soc. 59, 1918 (1963), ibid. 51, 193 (1963), ibid. 59, 1941 (1963); A. Katchalsky and P.F. Curran, "Non-equilibrium Thermodynamics in Biophysics", Harvard University Press, Cambridge, 1967.

7. J. Crank, "The Mathematics of Diffusion", $2^{\circ} \mathrm{Edit}$, Clarendon Press, Oxford, 1975. 
8. J. Crank and G.S. Park (edit.), "Diffusion in Polymers", Academic Press, N.Y. 1968.

9. H. Fujita, Adv. in Polymer Sci. 3, 1 (1961), H. Fujita, A. Kishimoto and K. Matsumoto, Trans. Faraday Soc. $\underline{56}, 424$ (1960).

10. R.M. Barrer, "Diffusion in and through Solids", $2^{\circ}$ Edit., Cambridge University Press, London, 1951.

11. T. Alfrey, E.F. Gurnee and W.G. Lloyd, J. Polymer Sci. C12, 249 (1966).

12. G.S. Park, Transport in Polymer Films in "Characterization of Coatings: Physical Techniques, Part II (R.R. Myers and J.S. Long, Eds.), Marcel Dekker, New York, 1976, p. 473; J.S. Vrentas and J.L. Duda, AIChEJ 25, 1 (1979); C.H.M. Jaques, H.B. Hopfenberg and V. Stannett, in "Permeability of Plastic Films and Coatings to Gases, Vapors and Liquids" (H.B. Hopfenberg, Ed.) Plenum Press, New York, 1974, p. 73.

13. S.A. Stern and H.L. Frisch, Ann. Rev. Mater. Sci. 11, 523 (1981); H.L. Frisch and S.A. Stern, CRC Critical Reviews in Solid State and Materials Science, 11, 123 (1983).

14. T. Graham, Phil. Mag. 32, 401 (1866).

15. S. Von Wroblewski, Wied. Ann. Phys. $\underline{8}, 29$ (1879).

16. H.A. Daynes, Proc. Roy. Soc. A94, 286 (1920).

17. R.M. Barrer, Trans. Faraday Soc. 35, 628 (1939), R.M. Barrer and R.R. Fergusson, ibid. 54, 989 (1958), R. Ash, R.M. Barrer and J. Petropoulos, Br. J. Appl. Phys. 14, 854 (1963), R. Ash, R.M. Barrer and D.G. Palmer, ibid. 16,873 (1965).

18. H.L. Frisch, J. Chem. Phys. 37, 2408 (1962).

19. H.L. Frisch, J. Phys. Chem. 61, 93 (1957), H. Pollak and H.L. Frisch, ibid. 63, 1022 (1959), H.L. Frisch, J. Chem. Phys. 36, 510 (1962).

20. S. Prager, J. Chem. Phys. 33, 122 (1960), see also the excellent review articles, and references cited therein, in "Mathematics and Physics of Disordered Materials", Springer Lecture Notes in Mathematics, Springer, Berlin, 1983 by B. Hughes and S. Prager and G.H. Malone, S.Y. Suh and S. Prager.

21. W. Strieder and R. Aris, "Variational Methods Applied to Problems of Diffusion and Reaction", Springer, Berlin, 1973.

22. H.L. Frisch and S. Prager, J. Chem. Phys. 54, 1451 (1971).

23. H.L. Frisch and J. Bdzil, J. Chem. Phys. 62, 4804 (1975). 
24. V. Stannett, H.B. Hopfenberg and J.H. Petropoulos, in "MTP International Review of Science", Vol. 8, Macromolecular Science, C.E.H. Bawn, Ed., Butterworths, London, 1972, p. 239; see also the articles in Polymer Eng. and Science, 20, No. 1 (1980) edited by D.R. Paul.

25. H.L. Frisch and C. Rogers, J. Chem. Phys. 40, 2293 (1964), H.L. Frisch and R. Volpe, J. Polymer Sci. (Phys. Ed.) 22, 2279 (1984).

26. W.R. Vieth, J.M. Howell and J.H. Hsieh, J. Membrane Sci. 1, 177, (1976), J.H. Petropoulos, J. Polymer Sci. A2, 1797 (1970), D.R. Paul and W.J. Koros, J. Polymer Sci. (Phys. Ed.) 14, 675 (1976), ibid. 16, 1947 and 2171 (1978), S.A. Stern and V. Saxena, J. Membrane Sci. I, 47 (1980); S.A. Stern and S. Trohilaki in "Barrier Polymers and structures" (ed. by W.J. Koros) ACS Symposium Series 423, Washington, D.C., 1990 .

27. T.T. Wang, T.K. Kwei and H.L. Frisch, J. Polymer Sci. A2, 7, 2019 (1969), ibid. I, 879 (1969); T.K. Kwei, T.T. Wang and H.M. Zupko, Macromolecules 5,645 (1972).

28. J. Jäckle and H.L. Frisch, J. Polymer Sci. (Phys. Ed.) 23, 675 (1985); J. Jäckle and H.L. Frisch, J. Chem. Phys. 85, 1621 (1986).

29. C.J. Durning, J. Polymer Sci. (Phys. Ed.) 23, 1831 (1985), C.J. Durning and J.L. Spencer, J. Polymer Sci. Polymer Lett. Ed. 23, 171 (1985).

30. see e.g. D.N. Theodorou and U.W. Suter, Macromolecules 18,1467 (1985); D. Rigby and R.J. Roe, J. Chem. Phys. 87, 7285 (1987), ibid. 89, 5280 (1988); D. Rigby, A. Kloczkowski, J.E. Mark and R.J. Roe, Polymer Preprints, 30,23 (1989); V.M. Shah, S.A. Stern and P.J. Ludovice, Macromolecules, 22, 4660 (1989); S. Trohilaki, L.C. DeBolt, J.E. Mark and H.L. Frisch, ibid. 23, 813 (1990); H. Takeuchi and K. Okazaki, J. Chem. Phys. 92, 5643 (1990), H. Takeuchi, ibid. 93,2062 (1990). 